\title{
Modeling the interaction of resource flows in the foreign trade system
}

\author{
Pyotr Kurenkov ${ }^{1, *}$, Alexey Volodin ${ }^{2}$, Anastasia Safronova ${ }^{3}$, Diana Kakhrimanova ${ }^{4}, J_{\text {J }}$ ef \\ Majerčák ${ }^{5}$ \\ ${ }^{1}$ Department of Transport business management and intelligent systems, Russian University of \\ Transport, Moscow, Obraztsova str., 9, building 9, 127994. Russia \\ ${ }^{2}$ Department of Operation of Water Transport, Russian University of Transport, Moscow, Obraztsova \\ str., 9, building 9, 127994. Russia \\ ${ }^{3}$ Department of Innovative Entrepreneurship, Bauman Moscow State Technical University, Moscow, \\ Baumanskaja 5, 105005, Russia \\ ${ }^{4}$ Department of International Transport Management and Supply Chain Management, Russian \\ University of Transport, Moscow, Obraztsova str., 9, building 9, 127994, Russia \\ ${ }^{5}$ Faculty of Operation and Economics of Transport and Communications, Zilina, Univerzitná 8215/1, \\ 010 26, Republic of Slovakia
}

\begin{abstract}
The article investigates the relationship of flows of various types (transport, cargo, information, financial, energy, legal and others) between themselves and elements in the delivery system of foreign trade cargo. A hierarchical scheme of points of interaction of various types of flows is proposed. It is note that optimization of flow control will create more favorable conditions for rhythmic work and coordination of transportation with other modes of transport, and a correctly calculate supply of goods to ports and border crossings will provide an opportunity for the economical use of rolling stock. The solution to this problem at a new level has become possible due to the widespread introduction of digital information technologies. A model of the topology of their interaction has been develop with the definition of an integral quality indicator of a particular route or delivery option. An algorithm for assessing the interdependence of various types of elements, flows, legal ties and the degree of complexity of the structure of the system for the delivery of foreign trade goods.
\end{abstract}

\section{Introduction}

Freight owners are constantly face with a number of problems: on what basic conditions to conclude an agreement for the purchase or sale of goods, how to choose a transportation route, how to pay the minimum price for transportation, and others. It is know that the commercial success of a product or transport service depends on not only the ratio of price and quality, investment in advertising, but also on how well the delivery, payment, transportation and storage schemes are chose [1,2]. The advantages of an integrated, systemic or, as it is now customary to call, - a logistics approach to managing the movement of transport, cargo, information, financial and energy flows served as one of the reasons for

\footnotetext{
* Corresponding author: petrkurenkov@mail.ru
} 
the conquest of the Russian market by foreign goods in the export of Russian electricity, natural resources and the import of transport services (due to transportation of Russian goods by foreign ships and automobiles, as well as payment for services related to transportation through firms registered abroad).

\section{Materials and Methods}

The development of the theory and practice of transport and logistics infrastructure management is the subject of the works of such authors as Fedorenko, E., Kudlac S., Kurbatova A., Kurenkov P., Majercak, J.

Many works are devoted to the optimization of management of flow processes of enterprises in the transport industry, including the work of the following scientists: Babin, M., Lipatov A., Persianov V., Stefancova V.

Optimization of management of the flow processes of enterprises in the transport industry will create more favorable conditions for rhythmic work and coordination of transportation with other modes of transport, and a correctly calculated delivery of goods to ports and border crossings will provide an opportunity for economical use of rolling stock.

For this reason, the purpose of the study is to develop a model for assessing the interdependence of various resource flows in the delivery system of foreign trade goods.

During the research, the following research methods used system analysis, complex analysis, calculation and analytical methods, comparison method, methods of mathematical modeling, statistical and quantitative methods.

\section{Results}

In the process of reforming, the vertical of management of the transportation process and its constituent structural units underwent hierarchical and abbreviated changes in names, as a result of which, for convenience and completeness of perception [3, 4], Figure 1 shows the previous and existing abbreviations of structural units involved in the management of train and shunting operations on railway stations, sections, tracks, landfills, etc.

Based on the above, a hierarchical scheme of points of interaction of various types of flows is propose, including dispatch, information, situational and other control centers for freight traffic in the mixed message system, shown in Figure 1.

The entire structure of the system for the delivery of foreign trade cargo (SDFTG) can be represent as a set of elements $\mathrm{X}=$ loading station (port), consignor, cargo owner, railway lines, stations for disbanding or forming trains, unloading station (port), consignee, forwarder, etc.) and a set of flows (transport - $\mathrm{T}$, cargo - G, information - Y, financial - F, energy - E and legal ties - P) interacting with each other. In turn, each element, flow and legal connection can be categorize - attributed to one category or another [5, 6].

Each element or subsystem of the SDFTG must be assignee a dynamic array consisting of constant and variable parameters of the current forecast and archive states. The parameters are not only the normative and reference information of the rules for the carriage of goods, tariff guides and various standards, but also the functional properties of the elements, determined by customer passports, technical and administrative acts of the stations, the train formation plan, codes of customs of seaports, charters of enterprises, licenses, certificates, etc. $[7,8]$.

Each parameter characterizing the elements (performance of handling equipment, storage capacity, span length, availability of licenses or the right to provide various types of services in the field of transport business, the procedure for handling arriving and departing trains at 
stations, the procedure for delivering / cleaning wagons to / from the port, the right for payment of the tariff according to the 10-01 price list and at the rates of the tariff policy, the bank in which the current account of the subject of the regional customs administration is located, the basic conditions of foreign trade contracts, etc.) has its own functional purpose and affects both the state and functioning separately taken element or subsystem of SDFTG, and the order of interaction of elements with flows of various types and categories, flows of various types among themselves, elements and flows with legal connections - that is, on the state and functioning of SDFTG as a whole.

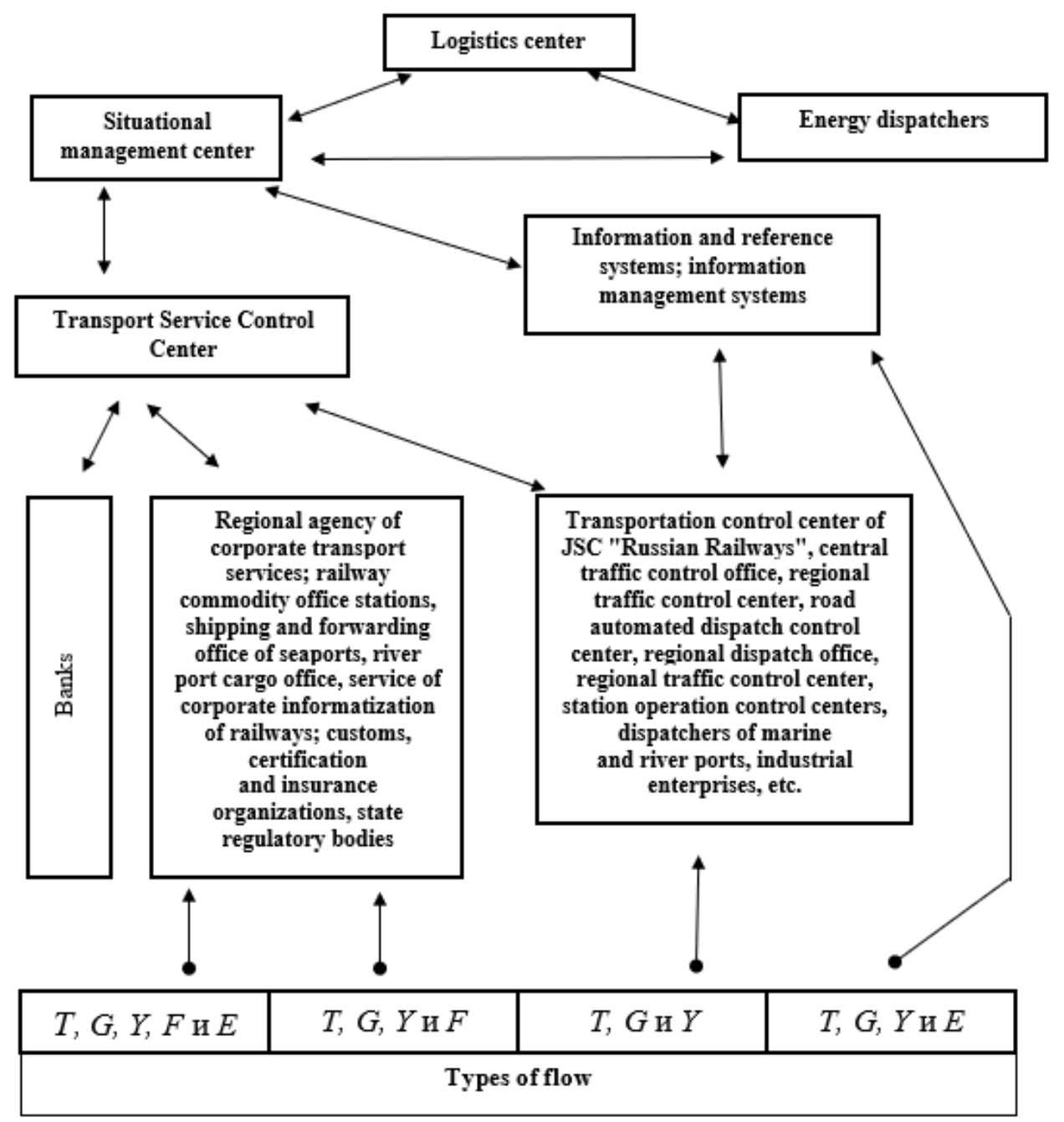

Fig. 1. Hierarchical diagram of points of interaction of various types of flows

One of the most important (qualitative) properties of SDFTG lies mainly in the strength of the connectivity of its elements, transport, cargo, information, financial and energy flows with a certain technology and legal basis for their interaction, or, in other words, this is the topological state space in which qualitative property of SDFTG $[9,10]$. It is a space that can be called the space of the structure of elements, structures of all types of flows and the structure of legal relations of various categories, since it is the structure of the relationship of elements, flows and the legal framework or individual subsystems of the SDFTG that 
determines its functioning. With a change (disappearance or weakening) of the connectivity between individual elements (closure of lines, natural disasters, wars, interstate conflicts, seizure of a current account, termination of sales and purchase agreements, forwarding contracts, chartering, declaration, revocation of licenses, power outages, etc. .) there is either the disappearance of SDFTG itself, or a change in its functional properties (quality).

Information about the qualitative state of SDFTG in statics given by its description in terms of incidence relations.

In general, a saturated linear plan in k-dimensional space is a collection of $\mathrm{M}+1$ points located in this space in such a way that they do not simultaneously belong to any subspace of dimension less than M. Such points define the vertices of a geometric figure that is call a k-dimensional simplex. A. Poincaré considered figures made up of simplexes (the so-called polyhedral) in the construction of homology theory - one of the sections of topology [11].

The notion "simplex" is formulate as follows: a simplex is the convex hull of linearly independent points in Euclidean space or a homeomorphism image; these points are call vertices of the simplex, and their number reduced by one is call the dimension of the simplex. Any subset of vertices of a simplex also defines a simplex - a face of the original simplex. Correct adjoining of simplexes in a polyhedron means that simplexes can intersect only along their common face.

However, according to computer terminology, there is also an excellent concept. In it is said, "between the vertices of the simplex, data can move in only one direction and it is impossible for the data flow to move in the opposite direction." In this case, only the information flow meant. In SDFTG, not only information flows, but all other types of flows move in both directions (exchange of information, return of incorrectly accrued sums of money, return of rolling stock to its owner, return of cargo to the departure station due to a commercial malfunction, etc.), therefore this the term for modeling the corresponding processes is unacceptable. The term "duplex", which characterizes the possibility of simultaneous transmission of data (movement of information flow) in both directions, is also not entirely appropriate, since between the same elements of the SDFTG and the subjects of regional customs departments (railway stations, ports, forwarders, etc.) move flows of various types. Therefore, the most suitable for formalizing interactions between elements, flows and legal ties in SDFTG seems to be the term "multiplex", which characterizes the movement of all types of flows in any direction, their interaction with elements, legal ties and among themselves [12,13].

An M-dimensional multiplex is define by its $M+1$ vertices $t_{1}, t_{2}, \ldots t_{a}, \ldots t_{l} ; g_{1}$, $g_{2}, \ldots, g_{b}, \ldots, g_{z} ; y_{1}, y_{2}, \ldots, y_{i}, \ldots, y_{m} ; f_{1}, f_{2}, \ldots, f c, \ldots, f h ; p_{1}, p_{2, \ldots}, p d, \ldots, p v$, etc., which are generic points. This means that they all do not lie in any of the $(M-1)$ - dimensional hyperactive planes.

To simulate the interaction of elements $\mathrm{x}_{i}$, flows $t_{a}, g_{b}, y_{i}, \mathrm{f}_{c}, \mathrm{e}_{w}$ and legal relations $p_{d}(x \in X, t \epsilon T, g \in G, y \in Y, f \epsilon F, e \epsilon E, p \in P)$ on products $X$ and $T, X$ and $G, X$ and $Y, X$ and $F, X$ and $E, X$ and $P$, as well as all the others, the relations $\lambda_{t}^{x} \in X \times T, \lambda_{g}^{x} \in X \times G$, $\lambda_{y}^{x} \in X \times Y, \lambda_{f}^{x} \in X \times F, \lambda_{e}^{x} \in X \times E, \lambda_{p}^{x} \in X \times P$, etc., which exist between the sets $X$ and $T, X$ and $G, X$ and $Y, X$ and $F, X$ and $E, X$ and $P$, etc. if and only if their constituent elements, flows and legal connections interact with each other. Moreover, if the interaction takes place, then $\lambda \geq 1$ (the values of $\lambda$ take depending on the accepted category of the element, flow and legal connection, otherwise they are equal to 0$)$. These ratios can be represent by incidence matrices $\left.\lambda_{t}^{x}=\left(\lambda_{i a}\right), \lambda_{g}^{x}=\left(\lambda_{i b}\right), \lambda_{y}^{x}=\left(\lambda_{i j}\right), \lambda_{f}^{x}=\left(\lambda_{i c}\right), \lambda_{e}^{x}=\left(\lambda_{i w}\right),\right), \lambda_{p}^{x}=\left(\lambda_{i d}\right)$, etc., where 


$$
\begin{aligned}
& \lambda_{i a}=1,2 \ldots N_{t}, \text { if }\left(x_{i}, t_{a}\right) \in \lambda_{t}^{x} ; \lambda_{i a}=0, i \text { if }\left(x_{i}, t_{a}\right) \notin \lambda_{t}^{x} ; \\
& \lambda_{i b}=1,2 \ldots N_{g}, \text { if }\left(x_{i} g_{b}\right) \in \lambda_{g}^{x} ; \lambda_{i b}=0, \text { if }\left(x_{i}, g_{b}\right) \notin \lambda_{g}^{x} ; \\
& \lambda_{i j}=1,2 \ldots N_{y}, \text { if }\left(x_{i}, y_{i}\right) \in \lambda_{y}^{x} ; \lambda_{i j}=0 \text {, if }\left(x_{i}, y_{i}\right) \notin \lambda_{y}^{x} ; \\
& \lambda_{i c}=1,2 \ldots N_{f}, \text { if }\left(x_{i}, f_{c}\right) \in \lambda_{f}^{x} ; \lambda_{i c}=0 \text {, if }\left(x_{i}, f_{c}\right) \notin \lambda_{f}^{x} ; \\
& \lambda_{i w}=1,2 \ldots N_{e}, \text { if }\left(x_{i}, e_{w}\right) \in \lambda_{e}^{x} ; \lambda_{i w}=0 \text {, if }\left(x_{i}, e_{w}\right) \notin \lambda_{e}^{x} ; \\
& \lambda_{i d}=1,2 \ldots N_{p}, \text { if }\left(x_{i}, p_{d}\right) \in \lambda_{p}^{x} ; \lambda_{i p}=0, \text { if }\left(x_{i}, p_{d}\right) \notin \lambda_{p}^{x} e t c .,
\end{aligned}
$$

where $N x, N t, N g, N y, N f, N e$ and $N p$ are the number of categories into which elements, flows and legal links are divide.

From a geometric point of view, the relationships $\lambda_{t}^{x}, \lambda_{g}^{x}, \lambda_{y}^{x}, \lambda_{f}^{x}, \lambda_{e}^{x}, \lambda_{p}^{x}$, etc. according to define simplifies complexes, denoted by $K_{x}(Y ; \lambda)$. We call them multiplicative complexes and denote them by $M_{x}^{t}\left(T ; \lambda_{t}^{x}\right), M_{x}^{g}\left(G ; \lambda_{g}^{x}\right), M_{x}^{y}\left(Y ; \lambda_{y}^{x}\right), M_{x}^{f}\left(F ; \lambda_{f}^{x}\right), \quad M_{x}^{e}\left(E ; \lambda_{e}^{x}\right)$, $M_{x}^{p}\left(F ; \lambda_{p}^{x}\right) e t c$

At the same time, each multiplex means a certain interaction of elements, flows of various types and legal relations between themselves (for example, "cargo shipment $\leftrightarrow$ ship", "transit car with processing $\leftrightarrow$ sorting station", "customs payments $\leftrightarrow$ bank", "navigator's receipt $\leftrightarrow$ fuel and energy complex "," a document confirming the export of goods $\leftrightarrow$ VAT refund "," agreement for the provision of information services $\leftrightarrow$ dislocation of a wagon or cargo "," energy flow $\leftrightarrow$ railway line "," set of shipping documents $\leftrightarrow$ commodity office ", etc.), and the totality of such multiplexes constitutes the structure of the entire SDFTG [14].

\begin{tabular}{|c|c|c|c|}
\hline Diret works & Relatios incidents & Incident matrices & Multiple complexes \\
\hline$X$ and $T$ & $\lambda t x \in X \times T$ & $\Lambda_{t}^{x}=\left(\lambda_{i a}\right)$ & $\operatorname{Mxt}(T ; \lambda t \boldsymbol{x})$ \\
\hline$X$ and $G$ & $\lambda \boldsymbol{g} \boldsymbol{x} \in \boldsymbol{X} \times \boldsymbol{G}$ & $\Lambda_{g}^{x}=\left(\lambda_{i b}\right)$ & $\operatorname{Mx} \boldsymbol{g}(\boldsymbol{G} ; \lambda \boldsymbol{g} \boldsymbol{x})$ \\
\hline$X$ and $Y$ & $\lambda \boldsymbol{y} \boldsymbol{x} \in \boldsymbol{X} \times \boldsymbol{Y}$ & $\Lambda_{y}^{x}=\left(\lambda_{i j}\right)$ & $\operatorname{Mxy}(Y ; \lambda y x)$ \\
\hline$X$ and $F$ & $\lambda \boldsymbol{f} \boldsymbol{x} \in \boldsymbol{X} \times \boldsymbol{F}$ & $\Lambda_{f}^{x}=\left(\lambda_{i c}\right)$ & $\operatorname{Mx} f(F ; \lambda f x)$ \\
\hline$T$ and $G$ & $\lambda \boldsymbol{g t} \in \boldsymbol{T} \times \boldsymbol{G}$ & $\Lambda_{g}^{t}=\left(\lambda_{a b}\right)$ & $\operatorname{Mtg}(G ; \lambda g t)$ \\
\hline$T$ and $Y$ & $\lambda \boldsymbol{y} \boldsymbol{t} \in \boldsymbol{T} \times \boldsymbol{Y}$ & $\Lambda_{y}^{t}=\left(\lambda_{a j}\right)$ & $\operatorname{Mty}(Y ; \lambda y t)$ \\
\hline$T$ and $F$ & $\lambda \boldsymbol{f t} \in \boldsymbol{T} \times \boldsymbol{F}$ & $\Lambda_{f}^{t}=\left(\lambda_{a c}\right)$ & $M t f(F ; \lambda f t)$ \\
\hline
\end{tabular}

Table 1 shows the designations of relations and incidence matrices, as well as multiplicative complexes for the corresponding products of element structures, flows and legal relationships.

Table 1. Notation of multiplicative complexes, relations and incidence matrices for the corresponding direct products of element structures, flows of various types and legal links

Multiple complexes, representing taken together SDFTG, are define as follows:

$1 . M_{x}^{t}\left(T ; \lambda_{t}^{x}\right), M_{x}^{g}\left(G ; \lambda_{g}^{x}\right), M_{x}^{y}\left(Y ; \lambda_{y}^{x}\right), M_{x}^{f}\left(F ; \lambda_{f}^{x}\right), M_{x}^{e}\left(E ; \lambda_{e}^{x}\right), M_{x}^{p}\left(P ; \lambda_{p}^{x}\right) e t c .$, in turn are, respectively, sets of multiplexes $\sigma_{s}^{t x} ; s=0,1, \ldots, N_{t}^{x} ; \sigma_{s}^{g x}, s=0,1, \ldots, N_{g}^{x} ; \quad \sigma_{s}^{y x}, s=$ $0,1, \ldots, N_{s}^{x} ; \sigma_{s}^{f x}, s=0,1, \ldots, N_{f}^{x} ; \sigma_{s}^{e x}, e=0,1, \ldots, N_{e}^{x} ; \sigma_{s}^{p x}, s=0,1, \ldots, N_{p}^{x}$ etc.

2. Each of the multiplexes $\sigma_{s}^{t x} \in M_{x}^{t}, \sigma_{s}^{g x} \in M_{x}^{g}, \sigma_{s}^{y x} \in M_{x}^{y}, \sigma_{s}^{f x} \in M_{x}^{f}, \sigma_{s}^{e x} \in M_{x}^{e}, \sigma_{s}^{p x} \in$ $M_{x}^{p}$, etc. are defined by some subsets of $(s+1)$ different $t_{a}, g_{b}, y_{i}, f_{c}, e_{w}$ and $p_{d}$, for each of which there is at least one $x_{m} \in X, t_{m} \in T, g_{m} \in G, y_{m} \in Y, f_{m} \in F, e_{m} \in E$ и $P_{m} \in P$, such that $\left(x_{m} ; t_{a}\right) \in \lambda_{t}^{x},\left(x_{m} ; g_{b}\right) \in \lambda_{g}^{x},\left(x_{m} ; y_{j}\right) \in \lambda_{y}^{x}, \quad\left(x_{m} ; f_{c}\right) \in \lambda_{f}^{x}, \quad\left(x_{m} ; e_{w}\right) \in \lambda_{e}^{x}$, $\left(x_{m} ; p_{d}\right) \in \lambda_{p}^{x}$ etc. for each of the $(s+1)$ values $t_{a}, g_{b}, y_{j}, f_{c}, e_{w}$ and $p_{d}$.

3. The multiplex $\boldsymbol{\sigma}_{\mathbf{0}}^{\boldsymbol{a}}$ is identified with $\boldsymbol{t}_{\boldsymbol{a}}, \boldsymbol{a}=\mathbf{1}, \ldots, \boldsymbol{l}$ (1 is the number of flows in the set $\mathrm{T}$ ); 
$\boldsymbol{\sigma}_{\mathbf{0}}^{\boldsymbol{b}}-\boldsymbol{c} \boldsymbol{g}_{\boldsymbol{b}}, \boldsymbol{b}=\mathbf{1}, \ldots, \boldsymbol{z}(z-$ is the number of flows in the set $\mathrm{G})$;

$\boldsymbol{\sigma}_{\mathbf{0}}^{\boldsymbol{j}}-\boldsymbol{c} \boldsymbol{y}_{\boldsymbol{j}}, \boldsymbol{j}=\mathbf{1}, \ldots, \boldsymbol{m}$ ( $m$ - is the number of flows in the set $\left.\mathrm{Y}\right)$;

$\boldsymbol{\sigma}_{\mathbf{0}}^{\boldsymbol{c}}-\boldsymbol{c} \boldsymbol{f}_{\boldsymbol{c}}, \boldsymbol{c}=\mathbf{1}, \ldots, \boldsymbol{h}(h-$ is the number of flows of the set $\mathrm{F})$;

$\boldsymbol{\sigma}_{\mathbf{0}}^{\boldsymbol{w}}-\boldsymbol{c} \boldsymbol{y}_{\boldsymbol{w}}, \boldsymbol{w}=\mathbf{1}, \ldots, \boldsymbol{u}$ ( $u$ is the number of flows of the set $\mathrm{E}$ );

$\boldsymbol{\sigma}_{\mathbf{0}}^{\boldsymbol{d}}-\boldsymbol{c} \boldsymbol{h}_{\boldsymbol{d}}, \boldsymbol{d}=\mathbf{1}, \ldots, \boldsymbol{p}(p-$ is the number of legal connections of the set $\mathrm{P})$.

4. Each subset of the multiplexes $\sigma_{s}^{t x}, \sigma_{s}^{g x}, \sigma_{s}^{y x}, \sigma_{s}^{f x}, \sigma_{s}^{e x}, \sigma_{s}^{p x}$, etc., defined by their $q^{t x}+$ $1, q^{g x}+1, q^{y x}+1, q^{f x}+1, q^{e x}+1, q^{p x}+1$, etc. vertices $\left(\left(q^{t x}<s^{t x}, q^{g x}<s^{g x}, q^{y x}<\right.\right.$ $s^{y x}, q^{f x}<s^{f x}, q^{e x}<s^{e x}, q^{p x}<s^{p x}$, etc.) are call $q^{q x}-, q^{g x}-, q^{y x}-, q^{f x}-, q^{e x}-$, $\boldsymbol{q}^{p \boldsymbol{x}}$-faces corresponding multiplexes and in turn form subsets $\boldsymbol{\sigma}_{q}^{t x} \in \boldsymbol{M}_{x}^{t}, \boldsymbol{\sigma}_{q}^{g x} \in \boldsymbol{M}_{x}^{g}, \boldsymbol{\sigma}_{q}^{y x} \in$ $M_{x}^{y}, \sigma_{q}^{f x} \in M_{x}^{f}, \sigma_{q}^{e x} \in M_{x}^{e}, \sigma_{q}^{p x} \in M_{x}^{p}\left(\sigma_{q}^{t x}<\sigma_{s}^{t x}, \sigma_{q}^{g x}<\sigma_{s}^{g x}, \sigma_{q}^{y x}<\sigma_{s}^{y x}, \sigma_{q}^{f x}<\sigma_{s}^{\phi x}, \sigma_{q}^{e x}<\right.$ $\left.\sigma_{s}^{\varepsilon x}, \sigma_{q}^{p x}<\sigma_{s}^{\pi x}\right)$.

The numbers $\boldsymbol{N}_{t}^{x}, \boldsymbol{N}_{g}^{x}, \boldsymbol{N}_{\boldsymbol{y}}^{x}, \boldsymbol{N}_{f}^{x}, \boldsymbol{N}_{\boldsymbol{e}}^{x}$ и $\boldsymbol{N}_{\boldsymbol{p}}^{x}$ determine the dimension of the multiplicative complexes $M_{t}^{x}\left(N_{t}^{x}=\operatorname{dim} M_{t}^{x}\right), \quad M_{g}^{x}\left(N_{g}^{x}=\operatorname{dim} M_{g}^{x}\right), \quad M_{y}^{x}\left(N_{y}^{x}=\operatorname{dim} M_{y}^{x}\right), \quad M_{f}^{x}\left(N_{f}^{x}=\right.$ $\left.\left.\operatorname{dim} M_{f}^{x}\right)\right), e^{x}\left(N_{e}^{x}=\operatorname{dim} M_{e}^{x}\right)$ and $M_{p}^{x}\left(N_{p}^{x}=\operatorname{dim} M_{p}^{x}\right)$, meaning the highest dimension for the respective multiplexes.

The sets $T, G, Y, F, E$ and $P$ are the sets of vertices of the multiplicative complexes $\boldsymbol{M}_{x}^{t}\left(\boldsymbol{T} ; \lambda_{t}^{x}\right), \boldsymbol{M}_{x}^{g}\left(\boldsymbol{G} ; \lambda_{g}^{x}\right), \boldsymbol{M}_{x}^{y}\left(\boldsymbol{Y} ; \lambda_{y}^{x}\right), \boldsymbol{M}_{x}^{f}\left(\boldsymbol{F} ; \lambda_{f}^{x}\right), \boldsymbol{M}_{x}^{e}\left(\boldsymbol{E} ; \lambda_{e}^{x}\right), \boldsymbol{M}_{x}^{p}\left(\boldsymbol{P} ; \lambda_{p}^{x}\right)$, etc.

Multiplexes $x_{i}$ (elements of SDFT) are convex polyhedral with vertices in Euclidean spaces $\boldsymbol{E}_{t}^{x}, \boldsymbol{E}_{g}^{x}, \boldsymbol{E}_{\boldsymbol{y}}^{\boldsymbol{x}}, \boldsymbol{E}_{f}^{x}, \boldsymbol{E}_{\boldsymbol{e}}^{\boldsymbol{x}}, \boldsymbol{E}_{\boldsymbol{p}}^{\boldsymbol{x}}$, etc., and multiplicative complexes $\boldsymbol{M}_{x}^{\boldsymbol{t}}\left(\boldsymbol{T} ; \lambda_{t}^{x}\right), \boldsymbol{M}_{x}^{g}\left(\boldsymbol{G} ; \lambda_{g}^{x}\right)$, $\boldsymbol{M}_{x}^{y}\left(\boldsymbol{Y} ; \lambda_{y}^{x}\right), \boldsymbol{M}_{x}^{f}\left(\boldsymbol{F} ; \lambda_{f}^{x}\right), \boldsymbol{M}_{x}^{e}\left(\boldsymbol{E} ; \lambda_{e}^{x}\right), \boldsymbol{M}_{x}^{p}\left(\boldsymbol{P} ; \lambda_{p}^{x}\right)$, etc. - a set of such polyhedral in the same spaces, the vertices of which are flows of various types and legal connections $\left(\boldsymbol{t}_{\boldsymbol{a}}, \boldsymbol{g}_{\boldsymbol{b}}, \boldsymbol{y}_{j}, \boldsymbol{f}_{\boldsymbol{c}}\right.$, $\boldsymbol{e}_{\boldsymbol{w}}$ и $\left.\boldsymbol{p}_{\boldsymbol{d}}\right)$. With this formulation, it is possible to assess the direct influence of the structures of elements, flows and legal ties on each other.

The ratio $\lambda^{-1}$ will give conjugate multiplicative complexes $\boldsymbol{M}_{t}^{x}\left(X ; \lambda_{t x}^{-1}\right), M_{g}^{x}\left(X ; \lambda_{g x}^{-1}\right)$, $\boldsymbol{M}_{y}^{x}\left(X ; \lambda_{y x}^{-1}\right), M_{f}^{x}\left(X ; \lambda_{f x}^{-1}\right), M_{e}^{x}\left(X ; \lambda_{e x}^{-1}\right), M_{p}^{x}\left(X ; \lambda_{p x}^{-1}\right)$, etc. Now, the incidence matrices for $\lambda_{t x}^{-1}, \lambda_{g x}^{-1}, \lambda_{y x}^{-1}, \lambda_{f x}^{-1}, \lambda_{e x}^{-1}, \lambda_{p x}^{-1}$, etc. are matrices $\Lambda_{t x}^{T}, \Lambda_{g x}^{T}, \Lambda_{y x}^{T}, \Lambda_{f x}^{T}, \Lambda_{e x}^{T}, \Lambda_{p x}^{T}$, etc., obtained by transposing $\boldsymbol{\Lambda}_{t x}, \boldsymbol{\Lambda}_{g x}, \Lambda_{y x}, \Lambda_{f x}, \Lambda_{e x}, \Lambda_{p x}$, etc. In this case, the reverse influence of the structures of elements, flows and legal connections on each other is studied.

Table 2 shows the designations of relations and transposed incidence matrices, as well as conjugate multiplicative complexes for the corresponding products of elements, flows of various types and legal relationships.

Table 2. Designation of conjugate multiplicative complexes, relations and incidence matrices for inverse products of structures elements, flows of various types and legal relationships

\begin{tabular}{|c|c|c|c|}
\hline Reverse works & Relatios incidents & $\begin{array}{l}\text { Incident } \\
\text { matrices }\end{array}$ & $\begin{array}{c}\text { Conjugate multiplicative } \\
\text { complexes }\end{array}$ \\
\hline Tи $X$ & $\lambda \boldsymbol{t} x-\mathbf{1} \in \boldsymbol{T} \times \boldsymbol{X}$ & $\Lambda_{t x}^{T}=\left(\lambda_{a i}\right)$ & $M_{t}^{x}\left(X ; \lambda_{t x}^{-1}\right)$ \\
\hline$G$ и $X$ & $\lambda \boldsymbol{g} \boldsymbol{x}-\mathbf{1} \in \boldsymbol{G} \times \boldsymbol{X}$ & $\Lambda_{g x}^{T}=\left(\lambda_{b i}\right)$ & $M_{g}^{x}\left(X ; \lambda_{g x}^{-1}\right)$ \\
\hline$Y$ и $X$ & $\lambda \boldsymbol{y} \boldsymbol{x}-\mathbf{1} \in \boldsymbol{Y} \times \boldsymbol{X}$ & $\Lambda_{y x}^{T}=\left(\lambda_{j i}\right)$ & $M_{y}^{x}\left(X ; \lambda_{y x}^{-1}\right)$ \\
\hline$F$ и $X$ & $\lambda \boldsymbol{f} x-\mathbf{1} \in \boldsymbol{F} \times \boldsymbol{X}$ & $\Lambda_{f x}^{T}=\left(\lambda_{c i}\right)$ & $M_{f}^{x}\left(X ; \lambda_{f x}^{-1}\right)$ \\
\hline$G$ и $T$ & $\lambda \boldsymbol{g t}-\mathbf{1} \in \boldsymbol{G} \times \boldsymbol{T}$ & $\Lambda_{g t}^{T}=\left(\lambda_{b a}\right)$ & $M_{g}^{t}\left(X ; \lambda_{g t}^{-1}\right)$ \\
\hline$Y$ и $T$ & $\lambda \boldsymbol{y t}-\mathbf{1} \in \boldsymbol{Y} \times \boldsymbol{T}$ & $\Lambda_{y t}^{T}=\left(\lambda_{j a}\right)$ & $M_{y}^{t}\left(X ; \lambda_{y t}^{-1}\right)$ \\
\hline$F$ и $T$ & $\lambda \boldsymbol{f t}-\mathbf{1} \in \boldsymbol{F} \times \boldsymbol{T}$ & $\Lambda_{f t}^{T}=\left(\lambda_{c a}\right)$ & $M_{y}^{t}\left(T ; \lambda_{f t}^{-1}\right)$ \\
\hline
\end{tabular}

Thus, the structure of the SDFTG is consider as consisting of 21 interacting complexes (21 direct and 21 reverse): complexes of elements, complexes of flows of various types transport, cargo, information, financial, energy and complexes of legal ties. 
Co-evolutionary interaction, considered from the point of view of its organization, is a chain link of conjugating complexes, which is characterize by asymmetry and the presence of a link. Breaking the bond in co-evolutionary interaction entails either disorganization or the emergence of separate independent complexes.

Let us consider some of the features of the use of multiplicative complexes for assessing the structure of SDFTG.

Multidimensional connected structure of multiplicative complexes $\boldsymbol{M}_{x}^{\boldsymbol{t}}\left(\boldsymbol{T} ; \boldsymbol{\lambda}_{\boldsymbol{t} x}\right)$ and $\boldsymbol{M}_{x}^{t}\left(X ; \lambda_{t x}^{-1}\right), \boldsymbol{M}_{x}^{g}\left(G ; \lambda_{g x}\right)$ and $\boldsymbol{M}_{g}^{x}\left(X ; \lambda_{g x}^{-1}\right), M_{x}^{y}\left(\boldsymbol{Y} ; \lambda_{y x}\right)$ and $\boldsymbol{M}_{y}^{x}\left(X ; \lambda_{y x}^{-1}\right)$ etc. can be studied in various ways using algebraic methods. For this, the concept of q-connection was introduced [15].

The concept of q-connectivity refers to the study of such bonds in the structure of the complexes $\boldsymbol{M}_{x}^{t}\left(\boldsymbol{T} ; \lambda_{t x}\right)$ and $\boldsymbol{M}_{t}^{x}\left(\boldsymbol{X} ; \lambda_{t x}^{-1}\right), \boldsymbol{M}_{x}^{g}\left(\boldsymbol{G} ; \lambda_{g x}\right)$ and $\boldsymbol{M}_{g}^{x}\left(\boldsymbol{X} ; \lambda_{g x}^{-1}\right), \boldsymbol{M}_{x}^{y}\left(\boldsymbol{X} ; \lambda_{y x}\right)$ and $M_{y}^{x}\left(X ; \lambda_{y x}^{-1}\right), M_{x}^{f}\left(F ; \lambda_{f x}\right)$ and $\boldsymbol{M}_{f}^{x}\left(X ; \lambda_{f x}^{-1}\right), M_{x}^{e}\left(E ; \lambda_{e x}\right)$ and $\boldsymbol{M}_{e}^{x}\left(X ; \lambda_{e x}^{-1}\right), M_{x}^{p}\left(P ; \lambda_{p x}\right)$ and $\boldsymbol{M}_{\boldsymbol{p}}^{\boldsymbol{x}}\left(\boldsymbol{X} ; \lambda_{\boldsymbol{p} x}^{-\mathbf{1}}\right)$ etc., for which each multiplex has a common vertex with neighboring multiplexes for $\quad q_{t}=0,1 \ldots, \operatorname{dim} M_{p}-1 ; \quad q_{g}=0,1 \ldots, \operatorname{dim} M_{g}-1 ; \quad q_{y}=$ $0,1 \ldots, \operatorname{dim} M_{y}-1 ; q_{f}=0,1 \ldots, \operatorname{dim} M_{f}-1 ; q_{e}=0,1 \ldots, \operatorname{dim} M_{e}-1$ and $q_{p}=$ $\mathbf{0}, \mathbf{1} \ldots, \boldsymbol{d i m} \boldsymbol{M}_{\boldsymbol{p}}-\mathbf{1}$. Geometrically, these links contain information about how the various types of elements, flows and legal links in SDFTG interact with each other. In other words, to represent multiplexes larger than $M_{t}=N_{t}, \operatorname{dim} M_{g}=N_{g}, \operatorname{dim} M_{y}=N_{y}, \operatorname{dim} M_{f}=$ $\boldsymbol{N}_{f y}, \quad \operatorname{dim} \boldsymbol{M}_{e}=\boldsymbol{N}_{e}$ и $\operatorname{dim} \boldsymbol{M}_{p}=\boldsymbol{N}_{\boldsymbol{p}}$. The complexes $\boldsymbol{M}_{x}^{t}\left(\boldsymbol{T} ; \lambda_{t x}\right)$ and $\boldsymbol{M}_{t}^{x}\left(X ; \lambda_{t x}^{-1}\right)$, $\boldsymbol{M}_{x}^{g}\left(G ; \lambda_{t x}\right)$ and $\boldsymbol{M}_{g}^{x}\left(\boldsymbol{X} ; \lambda_{g x}^{-1}\right), \boldsymbol{M}_{x}^{y}\left(\boldsymbol{Y} ; \lambda_{y x}\right)$ and $\boldsymbol{M}_{y}^{x}\left(\boldsymbol{X} ; \lambda_{y x}^{-1}\right), \boldsymbol{M}_{x}^{f}\left(\boldsymbol{F} ; \lambda_{f x}\right)$ and $\boldsymbol{M}_{f}^{x}\left(\boldsymbol{X} ; \lambda_{f x}^{-1}\right)$, $\boldsymbol{M}_{x}^{e}\left(E ; \lambda_{e x}\right)$ and $\boldsymbol{M}_{e}^{x}\left(E ; \lambda_{e x}^{-1}\right), \boldsymbol{M}_{x}^{p}\left(P ; \lambda_{p x}\right)$ and $\boldsymbol{M}_{\boldsymbol{p}}^{x}\left(\boldsymbol{X} ; \lambda_{p x}^{-1}\right)$ and all the others split into unconnected multiplexes at each level $\boldsymbol{q}_{\boldsymbol{t}}, \boldsymbol{q}_{\boldsymbol{g}}, \boldsymbol{q}_{\boldsymbol{y}}, \boldsymbol{q}_{\boldsymbol{f}}, \boldsymbol{q}_{\boldsymbol{e}}$ and $\boldsymbol{q}_{\boldsymbol{p}}$.

At multiplexes of the complexes $\boldsymbol{M}_{t}^{x}, \boldsymbol{M}_{g}^{x}, \boldsymbol{M}_{\boldsymbol{y}}^{x}, \boldsymbol{M}_{\boldsymbol{f}}^{x}, \boldsymbol{M}_{e}^{x}, \boldsymbol{M}_{\boldsymbol{p}}^{x}$ and etc. one can introduce equivalence relations, respectively $\gamma_{a}^{t x}, \gamma_{a}^{g x}, \gamma_{a}^{y x}, \gamma_{a}^{f x}, \gamma_{a}^{e x}, \gamma_{a}^{p x}$ and etc., defined as $\left(\sigma_{p}^{t x}, \sigma_{r}^{t x}\right) \in \gamma_{q}^{t x},\left(\sigma_{p}^{g x}, \sigma_{r}^{g x}\right) \in \gamma_{q}^{g x},\left(\sigma_{p}^{y x}, \sigma_{r}^{y x}\right) \in \gamma_{q}^{y x},\left(\sigma_{p}^{f x}, \sigma_{r}^{f x}\right) \in \gamma_{q}^{f x},\left(\sigma_{p}^{e x}, \sigma_{r}^{e x}\right) \in \gamma_{q}^{e x}$, $\left(\sigma_{p}^{p x}, \sigma_{r}^{p x}\right) \in \gamma_{q}^{p x}$, etc. if and only if the multiplexes $\sigma_{p}^{t x}, \sigma_{p}^{g x}, \sigma_{p}^{y x}, \sigma_{p}^{f x}, \sigma_{p}^{e x}, \sigma_{p}^{p x}$, etc. qrelated respectively to $\sigma_{r}{ }^{t x}, \sigma_{r}{ }^{g x}, \sigma_{r}{ }^{y x},{\sigma_{r}}^{f x},{\sigma_{r}}^{e x},{\sigma_{r}}^{p x}$, etc. The sets of all classes of equivalence relations $\gamma_{q}^{t x}, \gamma_{q}^{g x}, \gamma_{q}^{y x}, \gamma_{q}^{f x}, \gamma_{q}^{e x}, \gamma_{q}^{p x}$, etc. form the quotient sets $\boldsymbol{M}_{t}^{x} / \gamma_{q}^{t x}$, $\boldsymbol{M}_{g}^{x} / \gamma_{\boldsymbol{q}}^{g x}, \boldsymbol{M}_{y}^{x} / \gamma_{\boldsymbol{q}}^{y x}, \boldsymbol{M}_{f}^{x} / \gamma_{q}^{f x}, \boldsymbol{M}_{e}^{x} / \gamma_{q}^{e x}, \boldsymbol{M}_{\boldsymbol{p}}^{x} / \gamma_{\boldsymbol{q}}^{p x}$, etc., respectively, which define the partition of pairs of multiplicative complexes $\boldsymbol{M}_{x}^{t}\left(\boldsymbol{T} ; \gamma_{t x}\right)$ и $\boldsymbol{M}_{t}^{x}\left(\boldsymbol{X} ; \boldsymbol{\gamma}_{\boldsymbol{t x}}^{-1}\right)$, as well as all the others into pairwise disjoint subsystems (dividing the structure of the SDFTG into separate subsystems of elements, flows of various types and legal relationships, as well as pairs of flows of various types, etc.) called the classes of equivalence relations $Q_{q}^{t x}, Q_{q}^{g x}, Q_{q}^{y x}, Q_{q}^{f x}, Q_{q}^{e x}, Q_{q}^{p x}$, etc.

Multiplexes connected by a common vertex, for which $\boldsymbol{q}_{x}^{\boldsymbol{t}}=\mathbf{0}, \boldsymbol{q}_{x}^{\boldsymbol{b}}=\mathbf{0}, \boldsymbol{q}_{x}^{y}=\mathbf{0}, \boldsymbol{q}_{x}^{f}=\mathbf{0}$, $\boldsymbol{q}_{x}^{e}=\mathbf{0}, \boldsymbol{q}_{x}^{p}=\mathbf{0}$ form, respectively, sets of multiplexes of classes $\boldsymbol{Q}_{\mathbf{0}}^{t}, \boldsymbol{Q}_{\mathbf{0}}^{g}, \boldsymbol{Q}_{\mathbf{0}}^{\boldsymbol{y}}, \boldsymbol{Q}_{\mathbf{0}}^{f}, \boldsymbol{Q}_{\mathbf{0}}^{e}, \boldsymbol{Q}_{\mathbf{0}}^{p}$. And the multiplexes for which $\boldsymbol{q}_{x}^{t}=1, q_{x}^{g}=1, q_{x}^{y}=1, q_{x}^{f}=1, q_{x}^{e}=1, q_{x}^{p}=1$ are of the classes $Q_{1}^{t x}, Q_{1}^{g x}, Q_{1}^{y x}, Q_{1}^{f x}, q_{x}^{f}=1, Q_{1}^{e x}$, etc. 
Table 3. Faces of multiplexes, classes of equivalence relations, factor sets of multiplicative complexes

\begin{tabular}{|c|c|c|c|c|c|}
\hline \multicolumn{2}{|c|}{ Multiple complexes } & \multirow{2}{*}{$\begin{array}{l}\text { Facets the } \\
\text { relevant } \\
\text { multiplexes }\end{array}$} & \multirow{2}{*}{$\begin{array}{l}\text { Equivalenc } \\
\text { e relations }\end{array}$} & \multirow{2}{*}{ Factor sets } & \multirow{2}{*}{$\begin{array}{c}\text { Classes of } \\
\text { Equivalence } \\
\text { Relations }\end{array}$} \\
\hline Direct & Conjugate & & & & \\
\hline$M_{x}^{t}\left(T ; \lambda_{t}^{x}\right)$ & $M_{t}^{x}\left(X ; \lambda_{t x}^{-1}\right)$ & $\boldsymbol{\sigma}_{\boldsymbol{a}}^{\boldsymbol{x}}$ and $\boldsymbol{\sigma}_{\boldsymbol{i}}^{\boldsymbol{t}}$ & $\gamma_{q}^{t x}$ & $M_{t}^{x} / \gamma_{q}^{t x}$ & $Q_{q}^{t x}$ \\
\hline$M_{x}^{g}\left(G ; \lambda_{g}^{x}\right)$ & $M_{g}^{x}\left(X ; \lambda_{g x}^{-1}\right)$ & $\boldsymbol{\sigma}_{\boldsymbol{b}}^{\boldsymbol{x}}$ and $\boldsymbol{\sigma}_{\boldsymbol{t}}^{\boldsymbol{g}}$ & $\gamma_{q}^{g x}$ & $M_{g}^{x} / \gamma_{q}^{g x}$ & $Q_{q}^{g x}$ \\
\hline$M_{x}^{y}\left(Y ; \lambda_{y}^{x}\right)$ & $M_{y}^{x}\left(X ; \lambda_{y x}^{-1}\right)$ & $\sigma_{j}^{x}$ and $\sigma_{t}^{y}$ & $\gamma_{q}^{y x}$ & $M_{y}^{x} / \gamma_{q}^{y x}$ & $Q_{q}^{y x}$ \\
\hline$M_{x}^{f}\left(F ; \lambda_{f}^{x}\right)$ & $M_{f}^{x}\left(X ; \lambda_{f x}^{-1}\right)$ & $\boldsymbol{\sigma}_{\boldsymbol{c}}^{\boldsymbol{x}}$ and $\boldsymbol{\sigma}_{\boldsymbol{i}}^{f}$ & $\gamma_{q}^{f x}$ & $M_{f}^{x} / \gamma_{q}^{f x}$ & $Q_{q}^{f x}$ \\
\hline$M_{t}^{g}\left(G ; \lambda_{g}^{t}\right)$ & $M_{g}^{t}\left(T ; \lambda_{g t}^{-1}\right)$ & $\overline{\sigma_{b}^{t}}$ and $\boldsymbol{\sigma}_{\boldsymbol{a}}^{g}$ & $\gamma_{q}^{g t}$ & $M_{g}^{t} / \gamma_{q}^{g t}$ & $Q_{q}^{g t}$ \\
\hline$M_{t}^{y}\left(Y ; \lambda_{y}^{t}\right)$ & $M_{y}^{t}\left(T ; \lambda_{y t}^{-1}\right)$ & $\boldsymbol{\sigma}_{j}^{t}$ and $\sigma_{a}^{y}$ & $\gamma_{q}^{y t}$ & $M_{y}^{t} / \gamma_{q}^{y t}$ & $Q_{q}^{y t}$ \\
\hline$M_{t}^{f}\left(F ; \lambda_{f}^{t}\right)$ & $M_{f}^{t}\left(T ; \lambda_{f t}^{-1}\right)$ & $\sigma_{c}^{t}$ and $\sigma_{a}^{y}$ & $\gamma_{q}^{f t}$ & $M_{f}^{t} / \gamma_{q}^{f t}$ & $Q_{q}^{f t}$ \\
\hline
\end{tabular}

It should be not that if two multiplexes are $q$-linked, then they are also $q-1, \boldsymbol{q}-\mathbf{2}, \ldots, \mathbf{0}$ - linked in the SDFTG. Consequently, the conjugate complexes $M_{x}^{t}\left(T ; \lambda_{t x}\right)$ and $M_{x}^{t}\left(T ; \lambda_{t x}^{-1}\right)$, as well as all the others, can be considered as consisting of subsets of multiplexes of the same dimension having common faces. In other words, for each value of dimensions $q_{t}^{x}=$ $0,1, \ldots, \operatorname{dim} M_{t}^{x}, q_{g}^{x}=0,1, \ldots, \operatorname{dim} M_{g}^{x}, \quad q_{y}^{x}=0,1, \ldots, \operatorname{dim} M_{y}^{x}, q_{f}^{x}=0,1, \ldots, \operatorname{dim} M_{f}^{x} \quad q_{e}^{x}=$ $0,1, \ldots, \operatorname{dim} M_{e}^{x}$ и $q_{p}^{x}=0,1, \ldots, \operatorname{dim} M_{p}^{x}$ one can define a number different equivalence classes $Q^{x}, Q_{q}^{t t}, Q_{q}^{g}, Q_{q}^{y}, Q_{q}^{f}, Q_{q}^{e}$ and $Q_{q}^{p}$. This operation is call q-analysis of the SDFTG structure, and the vectors

$$
\begin{aligned}
& Q^{t x}=\left\{Q_{\text {dim } M}^{t x}, Q_{\text {dim } M-1}^{t x}, \ldots, Q_{1}^{t x}, Q_{0}^{t x}\right\}, \\
& Q^{g x}=\left\{Q_{\text {dim } M}^{g x}, Q_{\operatorname{dim} M-1}^{g x}, \ldots, Q_{1}^{g x}, Q_{0}^{g x}\right\}, \\
& Q^{y x}=\left\{Q_{\text {dim } M}^{y x}, Q_{\text {dim } M-1}^{y x}, \ldots, Q_{1}^{y x}, Q_{0}^{y x}\right\} \text {, } \\
& Q^{f x}=\left\{Q_{\text {dim } M}^{f x}, Q_{\operatorname{dim} M-1}^{f x}, \ldots, Q_{1}^{f x}, Q_{0}^{f x}\right\}, \\
& Q^{e x}=\left\{Q_{\text {dim } M}^{e x}, Q_{\operatorname{dim} M-1}^{e x}, \ldots, Q_{1}^{e x}, Q_{0}^{e x}\right\}, \\
& Q^{p x}=\left\{Q_{\text {dim } M}^{p x}, Q_{\operatorname{dim} M-1}^{p x}, \ldots, Q_{1}^{p x}, Q_{0}^{p x}\right\} \text { etc. }-
\end{aligned}
$$

- structural vectors, respectively, of elements, flows and legal relationships of various types.

If the sets $X$ and $T$ contain $n$ elements and $l$ flows, respectively, then the matrix $\Lambda_{t x}$ corresponds to a matrix of size $\boldsymbol{n}+\mathbf{1}$. Similarly, $\boldsymbol{\Lambda}_{\boldsymbol{g} x}$ is an $\boldsymbol{n} \times \boldsymbol{d}$ matrix, $\boldsymbol{\Lambda}_{\boldsymbol{y x}}$ is an $\boldsymbol{n} \times \boldsymbol{m}$ matrix, $\boldsymbol{\Lambda}_{\boldsymbol{f} x}$ is an $\boldsymbol{n} \mathbf{x} \mathbf{h}$ matrix, $\boldsymbol{\Lambda}_{\boldsymbol{e} x}$ is an $\boldsymbol{n} \mathbf{x} \mathbf{u}$ matrix, $\boldsymbol{\Lambda}_{\boldsymbol{p} x}$ is an $\boldsymbol{n} \mathbf{x} \mathbf{v}$ matrix, etc. consisting of zeros and numbers greater than or equal to one.

In the works $\Lambda_{t x} \Lambda_{t x}^{T}, \Lambda_{g x} \Lambda_{g x}^{T}, \Lambda_{y x} \Lambda_{y x}^{T}, \Lambda_{f x} \Lambda_{f x}^{T}, \Lambda_{e x} \Lambda_{e x}^{T}, \Lambda_{p x} \Lambda_{p x}^{T}$, etc. the numbers at the places $(\boldsymbol{i}, \boldsymbol{a}),(\boldsymbol{i}, \boldsymbol{b}),,(\boldsymbol{i}, \boldsymbol{j}),(\boldsymbol{i}, \boldsymbol{c}),(\boldsymbol{i}, \boldsymbol{w}),(\boldsymbol{i}, \boldsymbol{d})$, etc. are dot products, respectively rows $i$ and columns $a, b, j, c, w, d$ of matrices $\boldsymbol{\Lambda}_{t x}, \Lambda_{g x}, \Lambda_{y x}, \Lambda_{f x}, \Lambda_{e x}, \Lambda_{p x}$, etc. Therefore, they are equal to the values $\left(\boldsymbol{q}_{x}^{t}+\mathbf{1}\right),\left(\boldsymbol{q}_{x}^{g}+\mathbf{1}\right),\left(\boldsymbol{q}_{x}^{y}+\mathbf{1}\right),\left(\boldsymbol{q}_{x}^{f}+\mathbf{1}\right),\left(\boldsymbol{q}_{x}^{e}+\mathbf{1}\right)$, and $\left(\boldsymbol{q}_{x}^{p}+\mathbf{1}\right)$, in which $\boldsymbol{q}_{x}^{t}$, $\boldsymbol{q}_{x}^{\boldsymbol{g}}, \boldsymbol{q}_{x}^{\boldsymbol{y}}, \boldsymbol{q}_{x}^{\boldsymbol{f}}, \boldsymbol{q}_{x}^{\boldsymbol{e}}$ and $\boldsymbol{q}_{x}^{\boldsymbol{p}}$ are the dimensions of the common faces of the multiplexes $\boldsymbol{\sigma}_{\boldsymbol{p}}{ }^{\boldsymbol{t} x}$ and $\sigma_{r}^{t x}, \sigma_{p}{ }^{g x}$ and $\sigma_{r}{ }^{g x}, \sigma_{p}^{y x}$ and $\sigma_{r}^{y x},{\sigma_{p}}^{f x}$ and ${\sigma_{r}}^{f x}, \sigma_{p}{ }^{e x}$ and $\sigma_{r}{ }^{e x}, \sigma_{p}^{p x}$ and ${\sigma_{p}}^{p x} \sigma_{r}^{p x}$, etc.

So, the algorithm for assessing the connectivity of elements, flows and legal links in the structure of the SDFTG is as follows. To find the values $\boldsymbol{q}_{x}^{\boldsymbol{t}}, \boldsymbol{q}_{x}^{\boldsymbol{g}}, \boldsymbol{q}_{x}^{\boldsymbol{y}}, \boldsymbol{q}_{x}^{\boldsymbol{f}}, \boldsymbol{q}_{x}^{\boldsymbol{e}}, \boldsymbol{q}_{x}^{\boldsymbol{p}}$, etc. for common faces of all pairs $\boldsymbol{x}_{\boldsymbol{i}}$ - multiplexes in the multiplicative complexes $\boldsymbol{M}_{\boldsymbol{x}}^{\boldsymbol{t}}\left(\boldsymbol{T} ; \lambda_{\boldsymbol{t} \boldsymbol{x}}\right)$, $\boldsymbol{M}_{x}^{g}\left(\boldsymbol{G} ; \lambda_{g x}\right), \boldsymbol{M}_{x}^{y}\left(\boldsymbol{Y} ; \lambda_{y x}\right), \boldsymbol{M}_{x}^{f}\left(\boldsymbol{Y} ; \lambda_{f x}\right), \boldsymbol{M}_{x}^{e}\left(\boldsymbol{E} ; \lambda_{e x}\right)$, и $\boldsymbol{M}_{x}^{\boldsymbol{p}}\left(\boldsymbol{E} ; \lambda_{p x}\right)$, it is necessary: 
- create matrices $\Lambda_{t x} \Lambda_{t x}^{T}, \Lambda_{g x} \Lambda_{g x}^{T}, \Lambda_{y x} \Lambda_{y x}^{T}, \Lambda_{f x} \Lambda_{f x}^{T}, \Lambda_{e x} \Lambda_{e x}^{T}, \Lambda_{p x} \Lambda_{p x}^{T}$, etc. respectively sizes $n \times \boldsymbol{l}, \boldsymbol{n} \times \boldsymbol{d}, \boldsymbol{n} \times \boldsymbol{m}, \boldsymbol{n} \times \boldsymbol{h}, \boldsymbol{n} \times \boldsymbol{u}, \boldsymbol{n} \times \boldsymbol{v}$, etc., denoting them $\boldsymbol{\Omega}_{x}^{t}, \boldsymbol{\Omega}_{x}^{g}, \boldsymbol{\Omega}_{x}^{y}, \boldsymbol{\Omega}_{x}^{f}, \boldsymbol{\Omega}_{x}^{e}, \boldsymbol{\Omega}_{x}^{p}$ etc.;

- evaluate $\Lambda_{t x} \Lambda_{t x}^{T},-\Omega_{x}^{t}, \Lambda_{g x} \Lambda_{g x}^{T}-\Omega_{x}^{g}, \Lambda_{y x} \Lambda_{y x}^{T}-\Omega_{x}^{y}, \Lambda_{f x} \Lambda_{f x}^{T}-, \Omega_{x}^{f}, \Lambda_{e x} \Lambda_{e x}^{T}-\Omega_{x}^{e}, \Lambda_{p x} \Lambda_{p x}^{T}$ $-\boldsymbol{\Omega}_{x}^{p}$ etc.;

where $\boldsymbol{\Omega}_{x}^{t}=\left[\omega_{i \boldsymbol{a}}\right], \boldsymbol{\Omega}_{x}^{g}=\left[\omega_{i b}\right], \boldsymbol{\Omega}_{x}^{y}=\left[\omega_{i j}\right], \boldsymbol{\Omega}_{x}^{f}\left[\omega_{i c}\right], \boldsymbol{\Omega}_{x}^{w}=\left[\omega_{i w}\right], \boldsymbol{\Omega}_{x}^{p}=\left[\omega_{i d}\right]$ etc.

$$
\begin{aligned}
& \omega_{i a} \geq 1 \text { for } \boldsymbol{i}, \boldsymbol{a}=1,2, \ldots, n ; \\
& \omega_{i b} \geq 1 \text { for } \boldsymbol{i}, \boldsymbol{b}=1,2, \ldots, \boldsymbol{n} ; \\
& \omega_{i j} \geq 1 \text { for } \boldsymbol{i}, \boldsymbol{j}=1,2, \ldots, \boldsymbol{n} ; \\
& \omega_{i c} \geq 1 \text { for } \boldsymbol{i}, \boldsymbol{c}=1,2, \ldots, \boldsymbol{n} ; \\
& \omega_{i \boldsymbol{w}} \geq \mathbf{1} \text { for } \boldsymbol{i}, \boldsymbol{w}=\mathbf{1}, 2, \ldots, \boldsymbol{n} ; \\
& \omega_{i d} \geq 1 \text { for } \boldsymbol{i}, \boldsymbol{d}=1,2, \ldots, \boldsymbol{n} \text { etc. }
\end{aligned}
$$

Matrices $\boldsymbol{\Omega}_{x}^{t}, \boldsymbol{\Omega}_{x}^{g},, \mathbf{\Omega}_{x}^{y}, \boldsymbol{\Omega}_{x}^{f}, \boldsymbol{\Omega}_{x}^{e}$, etc. are call frequency relation matrices, symmetric with respect to the main diagonal. So $(\forall i, a)\left(\omega_{i a}=\omega_{a i}\right),(\forall i, b)\left(\omega_{i b}=\omega_{b i}\right),(\forall i, j)\left(\omega_{i j}=\right.$ $\left.\omega_{j i}\right),(\forall i, c)\left(\omega_{i c}=\omega_{c i}\right),(\forall i, w)\left(\omega_{i w}=\omega_{w i}\right),(\forall i, d)\left(\omega_{i d}=\omega_{d i}\right)$, etc.

Analysis for $M_{t}^{x}\left(X ; \lambda_{t x}^{-1}\right), \quad M_{g}^{x}\left(X ; \lambda_{g x}^{-1}\right), \quad M_{y}^{x}\left(X ; \lambda_{y x}^{-1}\right), \quad M_{f}^{x}\left(X ; \lambda_{f x}^{-1}\right), \quad M_{e}^{x}\left(X ; \lambda_{e x}^{-1}\right)$, $M_{p}^{x}\left(X ; \lambda_{p x}^{-1}\right)$, etc. is carry out by composing the matrices $\Lambda_{t x} \Lambda_{t x}^{T}-\Omega_{t}^{x}, \Lambda_{g x} \Lambda_{g x}^{T}-$ $\boldsymbol{\Omega}_{g}^{x}, \Lambda_{y x} \Lambda_{y x}^{T}-\Omega_{y}^{x}, \Lambda_{f x} \Lambda_{f x}^{T}-\Omega_{f}^{x}, \Lambda_{e x} \Lambda_{e x}^{T}-\Omega_{e}^{x}, \Lambda_{p x} \Lambda_{p x}^{T}-\Omega_{p}^{x}$, etc., where $\boldsymbol{\Omega}_{t}^{x}, \boldsymbol{\Omega}_{g}^{x}, \boldsymbol{\Omega}_{y}^{x}, \boldsymbol{\Omega}_{f}^{x}$, $\boldsymbol{\Omega}_{e}^{x}, \boldsymbol{\Omega}_{p}^{x}$, etc. are matrices, respectively, of the sizes $l \times l, d \times d, m \times m, h \times h, u \times u, v \times v$, etc., consisting of numbers greater than or equal to one.

To assess the qualitative (topological) properties of the SDFTG structure, an indicator is introduced - a "measure of complexity" of the structure - YSDFTG (M). Measures of the complexity of the interaction of structures of elements $-\Psi x(M)$, flows of various types $\Psi_{t}(M), \quad \Psi_{g}(M), \Psi_{y}(M), \Psi_{f}(M), \Psi_{e}(M)$ and legal connections $\Psi_{p}(M)$, are determined through the coordinates of the corresponding vectors $Q^{x}, Q^{t}, Q^{g}, Q^{y}, Q^{f}, Q^{e}$, and $Q^{p}$, according to the formulas given in Table 4 .

where $N_{x}, N_{t}, N_{g}, N_{y}, N_{f}, N_{e}, N_{p}$ etc. - dimensions of multiplicative complexes $\boldsymbol{M}_{x}, \boldsymbol{M}_{\boldsymbol{t}}, \boldsymbol{M}_{\boldsymbol{g}}, \boldsymbol{M}_{\boldsymbol{y}}, \boldsymbol{M}_{\boldsymbol{f}}, \boldsymbol{M}_{\boldsymbol{e}}, \boldsymbol{M}_{\boldsymbol{p}}$, equal to the highest values $\boldsymbol{q}^{t x}, \boldsymbol{q}^{g x}, \boldsymbol{q}^{y x}, \boldsymbol{q}^{\boldsymbol{f x}}, \boldsymbol{q}^{e x}$ and $\boldsymbol{q}^{p x}($ $N_{t}=\operatorname{dim} M_{t}, N_{g}=\operatorname{dim} M_{g}, N_{y}=\operatorname{dim} M_{y}, N_{f}=\operatorname{dim} M_{f}, N_{e}=\operatorname{dim} M_{e}$ and $N_{p}=$ $\left.\operatorname{dim} M_{p}\right)$;

where $N_{x}, N_{t}, N_{g}, N_{y}, N_{f}, N_{e}, N_{p}$, etc. are the dimensions of the multiplicative complexes $\boldsymbol{M}_{\boldsymbol{x}}, \boldsymbol{M}_{\boldsymbol{t}}, \boldsymbol{M}_{\boldsymbol{g}}, \boldsymbol{M}_{\boldsymbol{y}}, \boldsymbol{M}_{\boldsymbol{f}}, \boldsymbol{M}_{\boldsymbol{e}}, \boldsymbol{M}_{\boldsymbol{p}}$, etc., equal to the largest values $\boldsymbol{q}^{t x}, \boldsymbol{q}^{g x}, \boldsymbol{q}^{y x}, \boldsymbol{q}^{\boldsymbol{f x x}}, \boldsymbol{q}^{\boldsymbol{e x}}$ и $q^{e x}\left(N_{t}=\operatorname{dim} M_{t}, N_{g}=\operatorname{dim} M_{g}, N_{y}=\operatorname{dim} M_{y}, N_{f}=\operatorname{dim} M_{f}, N_{e}=\operatorname{dim} M_{e}\right.$ and $\left.N_{p}=\operatorname{dim} M_{p}\right)$;

$Q_{i}^{x}, Q_{a}^{t}, Q_{b}^{g}, Q_{j}^{y}, Q_{c}^{f}, Q_{w}^{e}$ and $Q_{d}^{p}$, - respectively, $i-t h, a-t h, b-t h, j-t h, c-t h, w-$ th, and $d-t h$, components, vectors $Q^{x}, Q^{t}, Q^{g}, Q^{y}, Q^{f}, Q^{e}$ and $Q^{p}$ from q-analysis. 
Table 4. Determination of the measure of the complexity of the interaction of structures of elements, flows and legal ties in SDFTG

\begin{tabular}{|c|c|}
\hline $\begin{array}{l}\text { A measure of the complexity of structures of } \\
\text { elements, flows } \\
\text { and legal ties when they influence each other }\end{array}$ & $\begin{array}{l}\text { A measure of the complexity of the } \\
\text { interaction of structures }\end{array}$ \\
\hline 1 & 2 \\
\hline$\Psi_{x}^{t}\left(M_{x}^{t}\right)=\frac{2\left\{\sum(i+1) * Q_{i}^{x t}\right\}}{\left[\left(N_{x}^{t}+1\right)\left(N_{x}^{t}+2\right)\right]}$ & \multirow[t]{2}{*}{$\Psi_{t x}(M)=\sqrt{\Psi_{x}^{\ddagger}(M)^{2}+\Psi_{x}^{\ddagger}(M)^{2}}$} \\
\hline$\Psi_{t}^{x}\left(M_{t}^{x}\right)=\frac{2\left\{\sum(i+1) * Q_{a}^{t x}\right\}}{\left[\left(N_{t}^{x}+1\right)\left(N_{t}^{x}+2\right)\right]}$ & \\
\hline$\Psi_{x}^{g}\left(M_{x}^{g}\right)=\frac{2\left\{\sum(i+1) * Q_{i}^{x g}\right\}}{\left[\left(N_{x}^{g}+1\right)\left(N_{x}^{g}+2\right)\right]}$ & \multirow[t]{2}{*}{$\Psi_{g x}(M)=\sqrt{\Psi_{x}^{g}(M)^{2}+\Psi_{g}^{x}(M)^{2}}$} \\
\hline$\Psi_{g}^{x}\left(M_{g}^{x}\right)=\frac{2\left\{\sum(b+1) * Q_{b}^{g x}\right\}}{\left[\left(N_{g}^{x}+1\right)\left(N_{g}^{x}+2\right)\right]}$ & \\
\hline$\Psi_{x}^{y}\left(M_{x}^{y}\right)=\frac{2\left\{\sum(i+1) * Q_{i}^{x y}\right\}}{\left[\left(N_{x}^{y}+1\right)\left(N_{x}^{y}+2\right)\right]}$ & \multirow[t]{2}{*}{$\Psi_{y x}(M)=\sqrt{\Psi_{x}^{y}(M)^{2}+\Psi_{y}^{x}(M)^{2}}$} \\
\hline$\Psi_{y}^{x}\left(M_{y}^{x}\right)=\frac{2\left\{\sum(j+1) * Q_{i}^{y x}\right\}}{\left[\left(N_{y}^{x}+1\right)\left(N_{y}^{x}+2\right)\right]}$ & \\
\hline$\Psi_{f}^{x}\left(M_{f}^{x}\right)=\frac{2\left\{\sum(c+1) * Q_{c}^{f x}\right\}}{\left[\left(N_{f}^{x}+1\right)\left(N_{f}^{x}+2\right)\right]}$ & \multirow[t]{2}{*}{$\Psi_{f x}(M)=\sqrt{\Psi_{x}^{f}(M)^{2}+\Psi_{f}^{\chi}(M)^{2}}$} \\
\hline$\Psi_{x}^{f}\left(M_{x}^{f}\right)=\frac{2\left\{\sum(i+1) * Q_{i}^{x f}\right\}}{\left[\left(N_{x}^{f}+1\right)\left(N_{x}^{f}+2\right)\right]}$ & \\
\hline$\Psi_{g}^{t}\left(M_{g}^{t}\right)=\frac{2\left\{\sum(b+1) * Q_{b}^{g t}\right\}}{\left[\left(N_{g}^{t}+1\right)\left(N_{g}^{t}+2\right)\right]}$ & \multirow[t]{2}{*}{$\Psi_{g t}(M)=\sqrt{\Psi_{g}^{t}(M)^{2}+\Psi_{t}^{g}(M)^{2}}$} \\
\hline$\Psi_{t}^{g}\left(M_{t}^{g}\right)=\frac{2\left\{\sum(a+1) * Q_{a}^{t g}\right\}}{\left[\left(N_{t}^{g}+1\right)\left(N_{t}^{g}+2\right)\right]}$ & \\
\hline$\Psi_{y}^{t}\left(M_{y}^{t}\right)=\frac{2\left\{\Sigma(j+1) * Q_{i}^{y t}\right\}}{\left[\left(N_{y}^{t}+1\right)\left(N_{y}^{t}+2\right)\right]}$ & \multirow[t]{2}{*}{$\Psi_{t y}(M)=\sqrt{\Psi_{y}^{t}(M)^{2}+\Psi_{t}^{y}(M)^{2}}$} \\
\hline$\Psi_{t}^{y}\left(M_{t}^{y}\right)=\frac{2\left\{\sum(a+1) * Q_{a}^{t y}\right\}}{\left[\left(N_{t}^{y}+1\right)\left(N_{t}^{y}+2\right)\right]}$ & \\
\hline$\Psi_{f}^{t}\left(M_{f}^{t}\right)=\frac{2\left\{\sum(c+1) * Q_{c}^{g t}\right\}}{\left[\left(N_{f}^{t}+1\right)\left(N_{f}^{t}+2\right)\right]}$ & \multirow[t]{2}{*}{$\Psi_{f t}(M)=\sqrt{\Psi_{f}^{t}(M)^{2}+\Psi_{t}^{f}(M)^{2}}$} \\
\hline$\Psi_{t}^{f}\left(M_{t}^{f}\right)=\frac{2\left\{\Sigma(a+1) * Q_{a}^{t f}\right\}}{\left[\left(N_{t}^{f}+1\right)\left(N_{t}^{f}+2\right)\right]}$ & \\
\hline
\end{tabular}

Thus, we obtain different vectors for the structure of elements $Q^{x}$, for the structures of flows $Q^{t}, Q^{g}, Q^{y}, Q^{f}, Q^{e}$ and for the structure of legal links $Q^{p}$. Calculating the complexity measures of the arrangement in the system of elements $\Psi_{x}(M)$, flows $\Psi_{t}(M), \Psi_{g}(M)$, 
$\Psi_{y}(M), \Psi_{f}(M), \Psi_{e}(M)$ and legal connections $\Psi_{p}(M)$ taking into account their interaction, quantitative characteristics of the quality of the SDFTG structure can be obtained.

Despite the fact that q-analysis is a rather effective approach for studying the degree of connectivity of SDFTG, nevertheless, it does not provide the necessary information about how important (integrated) each multiplex is in the overall structure of SDFTG. To take into account the individual properties of multiplexes, it is necessary to know the numerical value of the degree of integration of each individual multiplex in the structure of the entire SDFTG. The relative importance of this or that element, flow and legal connection in the general structure of SDFTG is characterize by their eccentricities.

In this regard, the following designations are introduce:

$\hat{\mathrm{g}}$ is the upper value of $q$ for $x_{i}$ or $t_{a}, g_{b}, y_{i}, f_{c}, e_{w}$ and $p_{d}$, i.e. $q=\operatorname{dim} M$;

$\breve{g}$ is the lower value of $q$ for $x_{i}$ or $t_{a}, g_{b}, y_{j}, f_{c}, e_{w}$, and $p_{d}$.

That is, $q$ is equal to the largest value of $q$ at which $x_{i}, t_{a}, g_{b}, y_{i}, f_{c}, e_{w}$, and $p_{d}$ are connected to some other multiplex, respectively, from the complexes $M_{x}^{t}\left(T ; \lambda_{t x}\right)$ and $M_{t}^{x}\left(X ; \lambda_{t x}^{-1}\right), M_{x}^{g}\left(G ; \lambda_{g x}\right)$ and $M_{g}^{x}\left(X ; \lambda_{g x}^{-1}\right), M_{g}{ }^{x}\left(X ; \lambda_{g x}{ }^{-1}\right), M_{x}^{f}\left(F ; \lambda_{f x}\right)$ and $M_{f}^{x}\left(X ; \lambda_{f x}^{-1}\right), M_{x}^{e}\left(E ; \lambda_{e x}\right)$ and $M_{e}^{x}\left(X ; \lambda_{e x}^{-1}\right), M_{x}^{p}\left(P ; \lambda_{p x}\right)$ and $M_{p}^{x}\left(X ; \lambda_{p x}^{-1}\right)$, as well as all others.

Then the eccentricities of individual multiplexes are determined as follows:

$$
\begin{aligned}
& \operatorname{Ecc}\left(x_{i}, t_{a}\right)=\frac{(\hat{\mathrm{g}}-\breve{\mathrm{g}})}{(\breve{\mathrm{g}}+1)} \\
& \operatorname{Ecc}\left(x_{i}, t_{a}\right)=\frac{(\hat{\mathrm{g}}-\breve{\mathrm{g}})}{(\breve{\mathrm{g}}+1)} \\
& \operatorname{Ecc}\left(\boldsymbol{x}_{\boldsymbol{i}}, \boldsymbol{g}_{\boldsymbol{h}}\right)=\frac{(\hat{\mathrm{g}}-\breve{\mathrm{g}})}{(\breve{\mathrm{g}}+\mathbf{1})} \\
& \operatorname{Ecc}\left(\boldsymbol{x}_{\boldsymbol{i}}, \boldsymbol{y}_{\boldsymbol{i}}\right)=\frac{(\hat{\mathrm{g}}-\breve{\mathrm{g}})}{(\breve{\mathrm{g}}+\mathbf{1})} \\
& \operatorname{Ecc}\left(\boldsymbol{x}_{\boldsymbol{i}}, \boldsymbol{f}_{\boldsymbol{i}}\right)=\frac{(\hat{\mathrm{g}}-\breve{\mathrm{g}})}{(\breve{\mathrm{g}}+\mathbf{1})} \\
& \operatorname{Ecc}\left(\boldsymbol{x}_{\boldsymbol{i}}, \boldsymbol{f}_{\boldsymbol{c}}\right)=\frac{(\hat{\mathrm{g}}-\breve{\mathrm{g}})}{(\breve{\mathrm{g}}+\mathbf{1})} \\
& \operatorname{Ecc}(x \boldsymbol{i}, \boldsymbol{e w})=\frac{(\hat{\mathrm{g}}-\breve{\mathrm{g}})}{(\breve{\mathrm{g}}+\mathbf{1})} \\
& \operatorname{Ecc}(x \boldsymbol{i}, \boldsymbol{p d})=\frac{(\hat{\mathrm{g}}-\breve{\mathrm{g}})}{(\breve{\mathrm{g}}+\mathbf{1})}
\end{aligned}
$$

The difference $\hat{\mathrm{g}}-\breve{\mathrm{g}}$ is a measure of the unusualness of any multiplex $\sigma$. Moreover, the equality $\hat{\mathrm{g}}-\breve{\mathrm{g}}=2$ is considered more significant if $q=1$, and not when $q=10$. Therefore, as the significance of eccentricities, we will use the above ratios (2), and not the absolute difference $\hat{\mathrm{g}}-\breve{\mathrm{g}}$. The values of eccentricities of various multiplexes make it possible to assess how much each element, stream or legal connection is integrate into the general structure of the SDFTG, that is, their significance in its functioning.

Table 5. the designations of structure vectors, frequency matrices of relations, matrix analysis of multiplicative complexes and eccentricities of multiplexes in these complexes are given. 
Table 5. Structural vectors, frequency matrices of relations and eccentricities of multiplexes in multiprimary complexes

\begin{tabular}{|c|c|c|c|}
\hline $\begin{array}{l}\text { Structural } \\
\text { vectors }\end{array}$ & $\begin{array}{c}\text { Frequency matrices } \\
\text { relations }\end{array}$ & $\begin{array}{c}\text { Matrix analysis of } \\
\text { complexes }\end{array}$ & $\begin{array}{r}\text { Eccentricities } \\
\text { multiplexes }\end{array}$ \\
\hline$Q^{t x}$ & $\boldsymbol{\Omega}_{x}^{t}(\forall \boldsymbol{i}, \boldsymbol{b})\left(\omega_{\boldsymbol{i} \boldsymbol{a}}=\omega_{\boldsymbol{a} i}\right)$ & $\Lambda_{t x} \Lambda_{t x}^{T}-\Omega_{x}^{f}$ & $\operatorname{Ecc}\left(x_{i}, t_{a}\right)$ \\
\hline$Q^{g x}$ & $\boldsymbol{\Omega}_{x}^{\boldsymbol{g}}(\forall \boldsymbol{i}, \boldsymbol{b})\left(\omega_{i \boldsymbol{b}}=\omega_{\boldsymbol{b} \boldsymbol{i}}\right)$ & $\Lambda_{g x} \Lambda_{g x}^{T}-\Omega_{x}^{g}$ & $\operatorname{Ecc}\left(x_{i}, g_{a b}\right)$ \\
\hline$Q^{y x}$ & $\boldsymbol{\Omega}_{x}^{\boldsymbol{y}}(\forall \boldsymbol{i}, \boldsymbol{j})\left(\omega_{i j}=\omega_{j i}\right)$ & $\Lambda_{y x} \Lambda_{y x}^{T}-\Omega_{x}^{y}$ & $\operatorname{Ecc}\left(x_{i}, y_{i}\right)$ \\
\hline$Q^{f x}$ & $\boldsymbol{\Omega}_{x}^{f}(\forall \boldsymbol{i}, \boldsymbol{c})\left(\omega_{i c}=\omega_{\boldsymbol{c} i}\right)$ & $\Lambda_{f x} \Lambda_{f x}^{T}-\Omega_{x}^{f}$ & $\operatorname{Ecc}\left(x_{i}, f_{c}\right)$ \\
\hline$Q^{g t}$ & $\boldsymbol{\Omega}_{t}^{g}(\forall \boldsymbol{a}, \boldsymbol{b})\left(\omega_{\boldsymbol{a b}}=\omega_{\boldsymbol{b a}}\right)$ & $\Lambda_{t g} \Lambda_{t g}^{T}-\Omega_{t}^{g}$ & $E c c\left(t_{a}, g_{b}\right)$ \\
\hline$Q^{y t}$ & $\boldsymbol{\Omega}_{t}^{y}(\forall \boldsymbol{a}, \boldsymbol{j})\left(\omega_{\boldsymbol{a j}}=\omega_{j \boldsymbol{a}}\right)$ & $\Lambda_{t y} \Lambda_{t y}^{T}-\Omega_{t}^{y}$ & $\operatorname{Ecc}\left(t_{a}, y_{i}\right)$ \\
\hline$Q^{f t}$ & $\boldsymbol{\Omega}_{\boldsymbol{t}}^{f}(\forall \boldsymbol{a}, \boldsymbol{c})\left(\omega_{\boldsymbol{a c}}=\omega_{\boldsymbol{c} \boldsymbol{a}}\right)$ & $\Lambda_{t f} \Lambda_{t f}^{T}-\Omega_{t}^{f}$ & $\operatorname{Ecc}\left(t_{a}, f_{c}\right)$ \\
\hline
\end{tabular}

\section{Discussion}

A measure of the complexity of the SDFTG structure can be determined for not only supply schemes, determining the degree of interaction of various types of elements and flows, but also for entities providing information, customs, forwarding, survey, warehouse, stevedoring and other services.

In the theory of the integrated operation of different types of transport, much attention was paid to the combined technology of freight transport in intermodal traffic, to a systematic analysis of the structure and technology of functioning of transport hubs, but mechanisms for changing the organizational structures or organizational forms of managing the system of delivery of foreign trade goods (SDFTG) were not considered. In this regard, this article proposed an algorithm for changing the organizational structures of management of the system for the delivery of foreign trade goods (SDFTG).

The proposed algorithm allows: firstly, to assess and analyze the structure of SDHTG with one integral quality indicator, and secondly, to determine the degree of integration of simplexes, duplexes, multiplexes, elements, flows, subsystems and individual structures into the general structure of SDFTG.

\section{References}

1. J. Majercak, S. Kudlac, M. Panak. Sustainable and Economically Efficient Five-point Supply Chain Management, in Proceedings of the 20th International Scientific Conference on Transport Means, 5-7 October 2016, Kaunas, Lithuania (2016)

2. O. Pokrovskaya, N. Reshetko, M. Kirpicheva, A. Lipatov, D. Mustafin The study of logistics risks in optimizing the company's transportation process. in IOP Conference Series: Materials Science and Engineering, 1-5 October 2019, Kislovodsk, Russia, 698, 6 (2019)

3. V. Persianov, E. Rudakova, A. Safronova, N. Pilipchuk. Trends in the Development of Corporate Ethics in the Company "DHL" and JSC "Russian Railways" in the Current Socio-Economic Conditions. in Advances in Intelligent Systems and Computing, 2227 May 2020, Novosibirsk, Russia, 2, 1116 (2020)

4. E. Majercakova, P. Majercak, J. Majercak. Logistics and New Trends in Supply Chain Management in Times of Economic Crisis, in 3rd International Conference on 
Education Reform and Management Innovation (ERMI 2015), 05-06 September 2015, Bangkok, Thailand, 78 (2015)

5. O. Pokrovskaya, R. Fedorenko, E. Khramtsova. Formation Of Transport And Storage Systems. International Scientific Conference "Global Challenges and Prospects of the Modern Economic Development". (GCPMED), 06 - 08 December 2018, Samara, Russia (2018)

6. A. Kurbatova, P. Kurenkov, A. Safronova, Yu. Tanaino. Selection of optimal supply schemes, in XII International Scientific Conference on Agricultural Machinery Industry, 10-13 September 2019, Rostov-on-Don, Russia (2019).

7. A. Egorov, N. Pilipchuk, I. Khmelev, V. Shatokhin, A. Kovkin. World experience in the development of container traffic, in IOP Conference Series: Materials Science and Engineering, 1-5 October 2019, Kislovodsk, Russia (2019)

8. P. Kurenkov, O. Pokrovskaya, M. Anastasov, M. Sokolov, A. Bochkov. Study of the current state of the transport infrastructure of road and rail transport of the Russian Federation, in IOP Conference Series: Materials Science and Engineering, 1-5 October 2019, Kislovodsk, Russia, 698, 6 (2019)

9. S. Kudlac, V. Stefancova, J. Majercak. Using the Saaty Method and the FMEA Method for Evaluation of Constraints in Logistics Chain, in 10th International Scientific Conference on Transportation Science and Technology. 04-05 May 2017, Vilnius, Lithuania, 187 (2017).

10. P. Hilton, S. Wiley. Homology Theory. Mir. Moscow. USSR (1966)

11. J. Casti. Large systems. Connectedness, complexity and catastrophes. Mir. Moscow. USSR (1982).

12. J. Majerčák, P. Majerčák. The impact of the dynamics of a train ride in a curve and its effect on a superstructure, in Transportation Research Procedia, 29-31 May 2019, Novy Smokovec, 40, (2019)

13. J. Gasparik, V. Luptak, P. Mesko, P. Kurenkov. Methodology for assessing transport connections on the integrated transport network. Komunikacie, 19, 2 (2017)

14. Ch. Hoare Interacting sequential processes. Mir. Moscow. USSR (1989) 